\title{
Primary Intraosseous Squamous Cell Carcinoma Arising from a Dentigerous Cyst of the Maxillary Wisdom Tooth
}

\author{
Hikaru Takahashi ${ }^{a}$ Yuichiro Takaku ${ }^{a}$ Ayako Kozakaia, b Hiroshi Otsuru ${ }^{a}$ \\ Yuya Murata $^{c}$ Michael W. Myers ${ }^{d}$ \\ aDepartment of Dentistry and Oral Surgery, National Hospital Organization, Tokyo Medical \\ Center, Tokyo, Japan; 'bepartment of Oral and Maxillofacial Surgery, Tokyo Dental College, \\ Tokyo, Japan; 'Department of Pathology, National Hospital Organization, Tokyo Medical \\ Center, Tokyo, Japan; IInternational Exchange Center, Showa University, Tokyo, Japan
}

\author{
Keywords \\ Primary intraosseous squamous cell carcinoma $\cdot$ Maxilla $\cdot$ Wisdom tooth
}

\begin{abstract}
The World Health Organization defines primary intraosseous squamous cell carcinoma (PIOSCC) as a squamous cell carcinoma (SCC) arising primarily within the jaws and having no connection with the oral mucosa. Here, we report a case of PIOSCC in which it was difficult to differentiate the condition from pericoronitis of an impacted maxillary wisdom tooth. The patient was a 27-year-old pregnant woman with a pain in the right maxillary wisdom tooth. The pain was diagnosed as pericoronitis of the right maxillary wisdom tooth, and the tooth was extracted under local anesthesia. During extraction, soft tissue was observed in the coronal part of the tooth, and it was diagnosed as SCC arising in a dentigerous cyst. Because the tumor may still be present, surgical resection was performed under general anesthesia. There has been no recurrence or metastasis at the 1-year follow-up. This case was histopathologically considered from being a benign odontogenic tumor to a malignant tumor. However, it revealed an extensive aberrant type and invasion equivalent to SCC. Thus, the histopathological diagnosis was PIOSCC arising from a dentigerous cyst. Although advanced cases of PIOSCC have been published, diagnosis of PIOSCC in the early stages is rare. In this case, we diagnosed pericoronitis of an impacted maxillary wisdom tooth and extracted the tooth. Therefore, we discovered it accidentally. In the early stages, diagnosis can be difficult both clinically and histopathologically.


Takahashi et al.: SCC Arising from a Dentigerous Cyst of the Maxilla

\section{Introduction}

According to the World Health Organization (WHO), primary intraosseous squamous cell carcinoma (PIOSCC) is defined as a squamous cell carcinoma (SCC) arising primarily within the jaws and having no connection with the oral mucosa [1]. It is believed to originate from the odontogenic epithelium, and its occurrence is rare. Here, we report a case of PIOSCC in which it was difficult to differentiate the condition from pericoronitis of an impacted maxillary wisdom tooth.

\section{Case Report}

A 27-year-old woman was referred to our department by her dentist in November 2017, because of a pain in the right maxillary wisdom tooth. The pain was diagnosed as pericoronitis of the wisdom tooth, and the woman was in the sixth month of pregnancy. The past and family histories were unremarkable.

At the time of her initial visit, the right maxillary wisdom tooth was completely impacted, with mild swelling of the mucosa, but there was no reddening. Panoramic radiography showed that the right maxillary wisdom tooth was impacted so that it might incline and rotate in a submerged orientation. There were no findings indicative of obvious transmission images in the coronal area, and no images showing irregular bone resorption (Fig. 1a). The pain was diagnosed as pericoronitis of the right maxillary wisdom tooth, and the tooth was extracted under local anesthesia. Extraction was performed by making an incision in the gingiva and forming a mucoperiosteal flap. Soft tissue was found in the coronal area of the wisdom tooth, and hence a curette was used to carefully separate the soft tissue from the surrounding tissue and verify the tooth. An elevator was used to extract the tooth, and the soft tissue was further separated from the surrounding tissue and excised. There was no obvious adhesion of the soft tissue to the surrounding tissue, and it was excised as a single mass (Fig. 1b). A tumorous lesion was suspected, and it was diagnosed as SCC arising from a dentigerous cyst. Histopathological findings indicated a tumor accompanied by keratinization, and the chromatin in the nuclei was condensed, with atypical mitotic figures observed occasionally (Fig. 2). There were well-differentiated fields demonstrating progression of keratinization, but there were also areas where stratified squamous epithelium was atypically sparse, and a transition area was noticed from the normal cyst wall epithelium to the atypical epithelium (Fig. 3a). On immunostaining, the atypical epithelium was positive for CK5/6, CK14, and p40. The rate of positivity for Ki-67 was around 10\%, but the majority of cells were positive for p53. Both normal and tumorous areas were partially positive for CK14 (Fig. 3b). In the normal squamous epithelium, results were negative for CK19, but tumorous areas were positive (Fig. 3c). It was judged that there was a strong possibility of positive tumor margins.

Because the patient was pregnant at the time, no computed tomography (CT) was done immediately after making a definitive diagnosis. Magnetic resonance imaging showed no infiltration of the tumor into the surrounding tissue, and no abnormalities were detected in the maxillary sinus. There was no enlargement of lymph nodes in the neck region. Extraction of the tooth had not been done keeping the tumor in mind, and because there was a possibility that the tumor still remained, consideration was given to additional excision. Because the patient was pregnant, the timing of the procedure was carefully considered. Given that we assumed that the tumor bulk had been excised, healing of the extraction site was progressing normally, and the magnetic resonance imaging revealed no obvious infiltration of the tumor into the surrounding area or metastasis into cervical lymph nodes, it was decided, after extensive discussion with the patient and her family, to perform additional excision after the patient had given birth. 
a

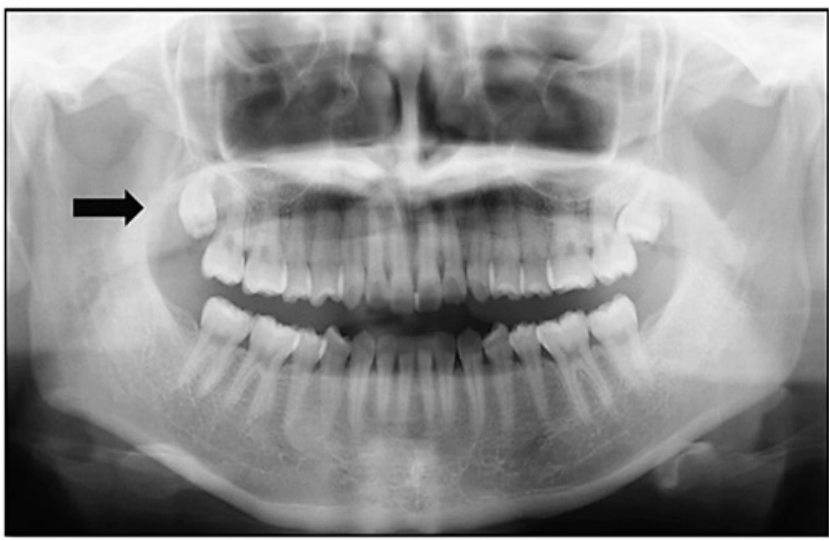

b

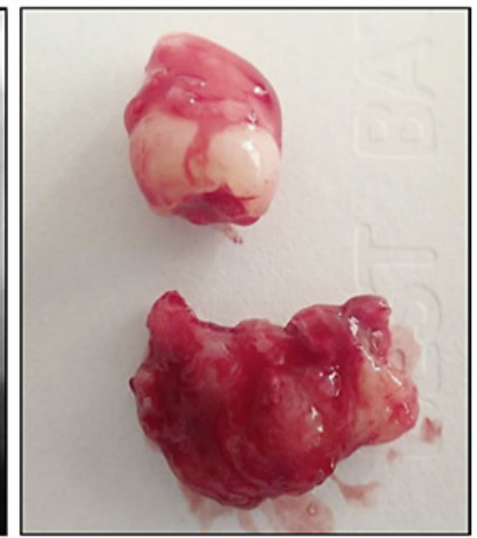

Fig. 1. Panoramic radiograph and extirpated material. a Panoramic radiograph showing a completely impacted maxillary tooth on the right side (arrow), but there are no findings that show transmission images or irregular bone resorption images in the coronal area. b Soft tissue was observed in the coronal area of the wisdom tooth, with no obvious adhesion to the surrounding tissue. It was possible to excise the soft tissue as a single mass.

a

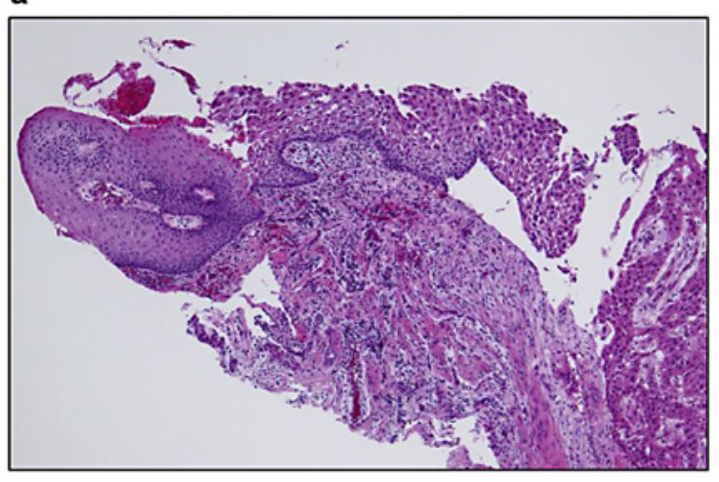

b

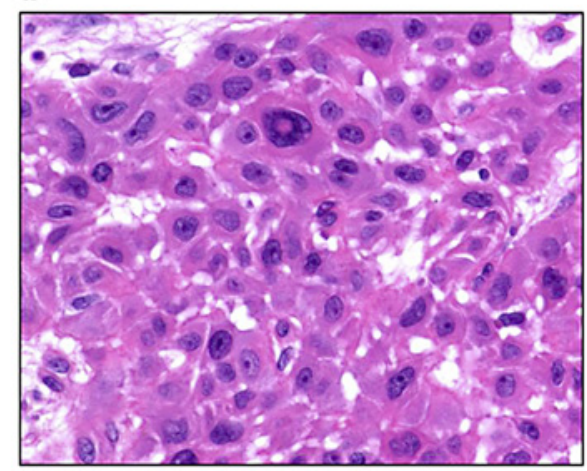

Fig. 2. Histopathological examination. a Histopathological image of the excised sample. HE staining. $\times 40$. b Histopathological image of the excised sample. HE staining. $\times 400$. The histopathological findings indicated a tumor accompanied by keratinization. The chromatin in the nuclei was condensed, with atypical mitotic figures observed occasionally.

After the patient had given birth, contrast-enhanced CT and positron emission tomography (PET)-CT were conducted, which demonstrated no indications of metastases to the cervical lymph nodes or distant metastases. Although the socket still remained where the tooth had been extracted, the mucous membrane of the socket was normal. In June 2018, a partial right maxillectomy was performed under general anesthesia. During the procedure, a mucoperiosteal flap was formed in the right maxillary molar region, and the surface of the bone was exposed. There was no irregular bone resorption on the surface of the maxillary 


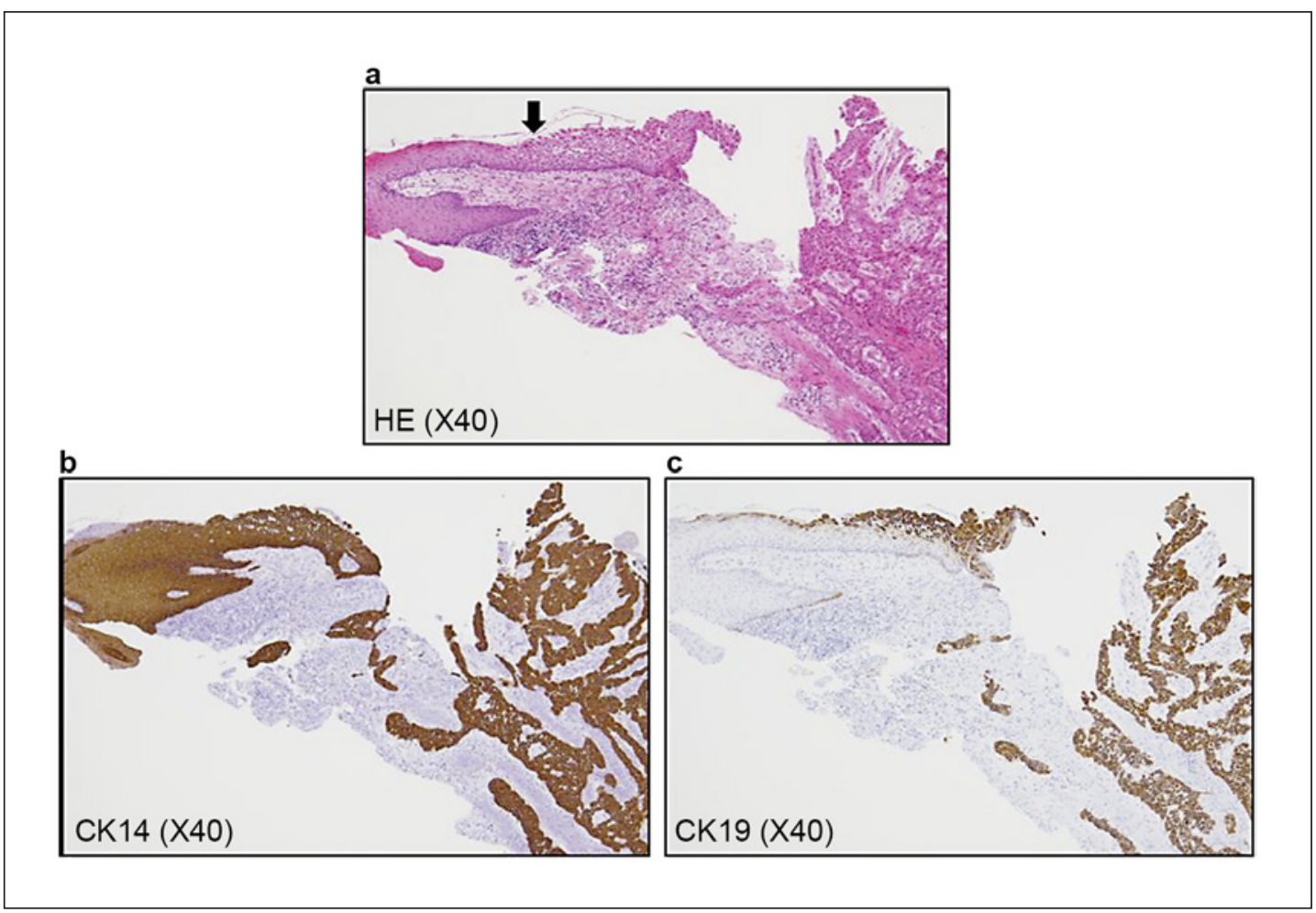

Fig. 3. Immunohistochemical findings. a Histopathological image of the excised sample. HE staining. $\times 40$. b Immunohistochemical findings of the excised sample. CK14 staining. $\times 40$. c Immunohistochemical findings of the excised sample. CK19 staining. $\times 40$. A transition area is observed between the normal cyst wall epithelium and the atypical epithelium. Arrow: transition section. The results are partially positive for CK14 in both the normal and the tumor area. CK19 staining is negative in the normal squamous epithelium but positive in tumor areas.

bone, and the surface of the bone was healthy. The maxillary second molar on the right side was included, and resection was performed from the distal side of the first molar on the anterior side to the pterygomaxillary on the posterior side, leaving one layer of bone in the bottom of the maxillary sinus in place on the upper side. One layer of the bone was removed from the transected surface, and the wound was sutured. The procedure was completed in $2 \mathrm{~h}$, and there was little bleeding. Histopathological examination of the excised sample showed no tumor remaining in the sample. PET-CT taken 8 months after the surgery revealed no recurrence nor metastasis. At the 1-year follow-up, there has been no recurrence or metastasis.

\section{Discussion}

According to the WHO, PIOSCC is classified as an odontogenic carcinoma arising within the jaws and having no initial connection with the oral mucosa. It is believed to originate in the odontogenic epithelial rest and is defined as SCC that has not metastasized from another organ $[1,2]$. Considering that it infiltrates the bone immediately from the early stages, and that clinical symptoms do not manifest easily, it is difficult to detect PIOSCC in the early stages, implying that the prognosis is generally not considered to be optimistic [3]. According to 
earlier reports, clinical symptoms include pain in the affected area, along with swelling and other indications of infection; however, in some cases, there are no obvious symptoms [4, 5]. In the early stages, the oral mucosa is normal, and in several cases, the first symptoms to appear include pain and slight looseness of the tooth, which tend to result in a diagnosis of periodontitis. Consequently, in several cases, periodontal treatment and extraction are implemented, but the healing is poor, and the carcinoma is often discovered after some time has elapsed [6]. As the condition progresses, symptoms such as bulging or swelling of the mandible and paresthesia caused by infiltration into the nerves occur, and a diagnosis can sometimes be made at this stage based on imaging [5]. Because of these characteristics, diagnosis can be difficult in the early stages, and by the time a diagnosis is made, in several cases, the tumor would have already infiltrated over a wide area [7,8]. In the present case, there were no clinical findings that differentiated the condition from pericoronitis of the wisdom tooth, making it difficult to suspect a tumorous lesion. On the panoramic radiograph, the wisdom tooth was impacted so that it might incline and rotate in a submerged orientation, which in hindsight was possibly due to the effects of the tumor. A definitive diagnosis is believed to be difficult, but it is important at all times to make a careful diagnosis based on imaging findings.

In terms of treatment, surgical excision is the first choice. The cancer is considered to have infiltrated into the mandible, and the most important consideration is to ensure that the tumor is thoroughly excised with the mandible, leaving an ample margin of safety.

Lesions that need to be histopathologically differentiated include ameloblastoma and ameloblastic carcinoma. According to an earlier report [9], the diagnosis of PIOSCC was made based on the following four conditions: (1) no abnormal findings were observed in the oral mucosa around the socket from which the tooth was extracted; (2) PET-CT images showed no findings suspicious of a primary lesion in the adjoining tissue or in remote organs, and thus the findings were negative for metastatic carcinoma; (3) histopathological testing showed no cystic ameloblastoma in the lesion; and (4) a transition of histopathologically normal cystic epithelium to SCC was confirmed. Confirmation of the area where the normal cystic epithelium transitions to SCC is believed to be difficult in some cases $[5,10]$, but in the present case, it was possible to histopathologically confirm the area where the normal cystic epithelium transitioned to SCC (Fig. 3a).

This report describes a case of PIOSCC in which it was difficult to differentiate the condition from pericoronitis of an impacted maxillary wisdom tooth. This case was considered histopathologically from a benign odontogenic tumor to a malignant tumor. However, it revealed an extensive aberrant type and invasion equivalent to SCC. Thus, the histopathological diagnosis was PIOSCC arising from a dentigerous cyst. Although advanced cases of PIOSCC have been published, cases diagnosed in the early stages are rare. In this case, we diagnosed pericoronitis of an impacted maxillary wisdom tooth and extracted the tooth. Therefore, we discovered it accidentally. In the early stages, diagnosis can be difficult both clinically and histopathologically.

\section{Acknowledgements}

The authors would like to thank Enago (www.enago.jp) for the English language review.

\section{Statement of Ethics}

Written ethical approval for the publication of the present case report was obtained from the patient. Since this is a case report, IRB approval was not needed. 


\section{Disclosure Statement}

The authors have no conflicts of interest to declare.

\section{Funding Sources}

No funding was received for this article, and the authors have no conflicts of interest directly relevant to this report.

\section{Author Contributions}

H.T. made substantial contributions to the concept and design of the study, as well as to data acquisition and interpretation; Y.T., A.K., and Y.M. were involved in drafting the manuscript and critical revision for important intellectual content; H.T. approved the final version of the manuscript that was submitted for publication. All authors read and approved the final manuscript.

\section{References}

1 Barnes L, Everson JW, Reichart P, Sidransky D, editors. World Health Organization classification of tumors. Pathology and genetics of head and neck tumors. Lyon: IARC Press; 2005. p. 290-1.

2 El-Naggar AK, Chan JKC, Grandis GR, Takata T, Slootweg PJ, editors. World Health Organization classification of head and neck tumours. Lyon: IARC Press; 2017. p. 207-9.

3 Thomas G, Pandey M, Mathew A, Abraham EK, Francis A, Somanathan T, et al . Primary intraosseous carcinoma of the jaw: pooled analysis of world literature and report of two new cases. Int J Oral Maxillofac Surg. 2001 Aug; 30(4):349-55.

4 Hino S, Tanaka H, Nakashiro K, Hamakawa H. Primary intraosseous squamous cell carcinoma derived from a dentigerous cyst. J Oral Maxillofac Surg Med Pathol. 2016 Jul;28(4):307-9.

5 Uchida K, Ochiai T, Sinohara A, Miki M, Muto A, Yoshinari N, et al. Primary intraosseous odontogenic carcinoma arising from a dentigerous cyst. J Hard Tissue Biol. 2013;22(3):375-82.

6 Fanibunda K, Soames JV. Malignant and premalignant change in odontogenic cysts. J Oral Maxillofac Surg. 1995 Dec;53(12):1469-72.

7 Bodner L, Manor E, Shear M, van der Waal I. Primary intraosseous squamous cell carcinoma arising in an odontogenic cyst: a clinicopathologic analysis of 116 reported cases. J Oral Pathol Med. 2011 Nov;40(10):733-8.

8 Woolgar JA, Triantafyllou A, Ferlito A, Devaney KO, Lewis JS, Rinaldo A, et al. Intraosseous carcinoma of the jaws: a clinicopathologic review. Part III: primary intraosseous squamous cell carcinoma. Head Neck. 2013 Jun;35(6):906-9.

9 Gardner AF. A survey of odontogenic cysts and their relationship to squamous cell carcinoma. Dent J. 1975 Mar;41(3):161-7.

10 Yasuoka T, Yonemoto K, Kato Y, Tatematsu N. Squamous cell carcinoma arising in a dentigerous cyst. J Oral Maxillofac Surg. 2000 Aug;58(8):900-5. 